\title{
Visuospatial encoding deficits and compensatory strategies in schizophrenia revealed by eye movement analysis during a working memory task
}

\author{
Cocchi L, Bosisio F, Berchtold A, Orita A, Debbané M, Wood SJ, \\ Schenk F. Visuospatial encoding deficits and compensatory strategies in \\ schizophrenia revealed by eye movement analysis during a working \\ memory task.
}

Objective: To investigate scanpath abnormalities during the encoding of static stimuli in schizophrenia and their interaction with visuospatial working memory (VSWM) dysfunction.

Methods: Outpatients with schizophrenia and control subjects were asked to encode a static pattern for subsequent recognition after a short delay. We measured the number of correct and incorrect choices. We also assessed the number and the distribution of fixations, the scanning time in specific regions of interest (ROIs) and the head movements during the encoding of the stimuli. The distributions of fixations and scanning time in definite ROIs during the discrimination of the correct pattern from the foils were also measured.

Results: Patients recognised fewer correct patterns than controls. Correct trials in patients were characterised by a specific exploration of the central part of the stimulus during its presentation, whereas this feature was absent in incorrect trials. However, the scanning time and the numbers of fixations and head movements during encoding were similar in both groups and unrelated to recognition accuracy. In both groups, correct trials were associated with a selective exploration of the correct pattern amongst the six possibilities during recognition. Furthermore, patients gave more attention to incorrect patterns with a leftmost element identical to that of the correct response and also those approximating its global structure.

Conclusion: Patients showed a VSWM deficit independent of oculomotor dysfunctions and head movements during encoding. Patients' correct trials were related to specific scanning during encoding and discrimination phases. Analysis of these patterns suggests that patients try to compensate for reduced VSWM ability by using specific encoding strategies.

\author{
Luca Cocchi ${ }^{1,2,3,4}$, Francesca \\ Bosisio $^{2}$, André Berchtold ${ }^{5}$, Alina \\ Orita $^{6}$, Martin Debbané 7 , Stephen \\ J. Wood ${ }^{1}$, Françoise Schenk ${ }^{2,3,8}$ \\ ${ }^{1}$ Melbourne Neuropsychiatry Centre, University of \\ Melbourne, c/o National Neuroscience Facility, \\ Melbourne, Australia; ${ }^{2}$ Institute of Psychology, \\ University of Lausanne, Switzerland; ${ }^{3}$ Center for \\ Psychiatric Neuroscience (CNP), Prilly, Switzerland; \\ ${ }^{4}$ Institute of Sport Science and Physical Education \\ (ISSEP), University of Lausanne, Switzerland; ${ }^{5}$ Institute \\ of Applied Mathematics (IMA), University of Lausanne, \\ Switzerland; ${ }^{6}$ University Psychiatric Adult Department \\ of Lausanne (DUPA), Centre Hospitalier Universitaire \\ Vaudois and University of Lausanne, Lausanne, \\ Switzerland; ${ }^{7}$ Service Médico-Pédagogique, University \\ of Geneva School of Medicine, Geneva, Switzerland; \\ and ${ }^{8}$ Department of Physiology, University of \\ Lausanne, Switzerland
}

Keywords: encoding; eye movement; fixation; scanpath; schizophrenia; strategy; visuospatial; working memory

Dr Luca Cocchi, Melbourne Neuropsychiatry Centre, The University of Melbourne,

c/o National Neuroscience Facility,

161 Barry Street, Carlton South 3053, Australia..

Tel: (+61 3) 8344 1861;

Fax: (+61 3) 9348 0469;

E-mail: Icocchi@unimelb.edu.au

\section{Introduction}

Visuospatial working memory (VSWM) is the process by which a limited amount of visuospatial information is maintained and manipulated for a short time. This ability is crucial to plan future behaviour but because of its limited capacity, the selection of relevant information is critical. It has been proposed that VSWM can regulate oculomotor processes in order to select or recognise relevant information in the visual field $(1-3)$.

Working memory dysfunctions in schizophrenia are well documented (4) and deficits in its visuospatial component have been proposed as an illness trait marker (5). They are accompanied by scanpath abnormalities during the exploration of geometrical figures (6) or images with socially salient content [e.g. faces; see (7-10)]. Patients show shorter scanpath lengths and fewer fixations on informative 


\section{Cocchi et al.}

parts of the scene $(9-16)$. This particular oculomotor behaviour in schizophrenia might be related to a general cognitive deficit in the selection, integration and use of relevant information, commonly detected in VSWM deficits. However, the spatial distribution of fixations during the encoding of complex visual stimuli in schizophrenia seems independent of stimulus type (e.g. natural scene vs. images with socially salient content). This suggests that basic oculomotor function can contribute to the abnormal ocular behaviour and eventually disturb subsequent global information processing (17).

The relationship between VSWM and oculomotor functions has been investigated in a number of studies (18-21). In spite of incongruent results, recent findings argue that basic anomalies in smooth pursuit eye movements (SPEM) are insufficient by themselves to explain VSWM deficits in patients with schizophrenia $(18,42)$. During the encoding of dynamic stimuli, SPEM involve complex processes (22), not directly comparable to those of scanpath generation (i.e. saccades and fixations). Therefore, the relationship between oculomotor and VSWM processes during a complex VSWM task presenting static stimuli in patients with schizophrenia requires further investigation.

Recently, we investigated VSWM in patients with schizophrenia using a new task (23). In this paradigm, subjects were asked to encode stimuli composed of single multi-segment line patterns for later recognition. After a short delay, they had to discriminate the original stimulus from five incorrect patterns that had some properties in common with the target. Recognition performance was reduced in participants with schizophrenia as compared to healthy controls. They expressed a preference for incorrect patterns with fragmentary encoding concentrated on the left or right part of the stimulus, or approximating its global shape. We interpreted these results as a general impairment in global encoding abilities, combining early stage visual processes (24-27) and VSWM dysfunctions (4). Early stage visual processes are needed to produce a fast, but imprecise, internal representation of the stimulus. VSWM functions assist encoding by orienting attention $(28,29)$ (and the eyes) on critical regions of the stimulus in order to complete the representation.

Behavioural results obtained in our previous study did not allow a clear assessment of the contribution of VSWM and oculomotor processes to the patients' impaired performances. In order to do this, we reproduced our initial investigation using the VSWM task and combined it with an analysis of the participants' exploratory eye movements during the encoding and the recognition phases. The present study involves an independent group of outpatients meeting diagnostic criteria for schizophrenia. It emphasised the following points: (a) comparison of the scanpath patterns of patients and control subjects during stimulus encoding in relation with subsequent choice efficacy, (b) detailed analysis of the scanpaths during stimuli discrimination in order to qualify the ability to build a coherent internal stimulus representation online and (c) assessment of a possible interaction between these two processes.

\section{Method}

Subjects

Sixteen individuals meeting diagnostic criteria for schizophrenia (4th ed., DSM-IV; American Psychiatric Association) and 16 controls entered this study. Patients with schizophrenia were recruited from the outpatient population of the Psychiatric Hospital of Cery, Lausanne (Switzerland). As shown in Table 1, patients and controls were carefully matched for sex, handedness and age. Individuals with a history of neurological or medical illness likely to affect the nervous system, traumatic brain injury and reduced, uncorrected, visual acuity were excluded. All subjects in the control group reported no history of substance or alcohol abuse and were free from psychiatric illness or symptoms, as investigated by a structured diagnostic psychiatric interview for DSMIV and ICD-10 [Mini-International Neuropsychiatric Interview, see (30)].

Participants were recruited in the region of Lausanne and provided with a complete description

Table 1. Sociodemographic information of 16 patients with schizophrenia and 16 healthy comparison subjects

\begin{tabular}{|c|c|c|c|}
\hline & Schizophrenia & Control & Statistic \\
\hline $\operatorname{Sex}(n)$ & $14 \mathrm{~m} ; 2 \mathrm{f}$ & 14 m; $2 f$ & \\
\hline Education, years (SD) & $12( \pm 1.7)$ & $14.5( \pm 2.5)$ & $\begin{array}{l}t(30)=3.227 \\
p=.003\end{array}$ \\
\hline Laterality (n right) & 13 & 12 & \\
\hline Age at testing, years (SD) & $43( \pm 10.5)$ & $41.6( \pm 10.6)$ & $\begin{array}{l}t(30)=0.390 \\
p=.70\end{array}$ \\
\hline \multicolumn{4}{|l|}{ Clinical information } \\
\hline $\begin{array}{l}\text { Psychiatric treatment, } \\
\text { years (SD) }\end{array}$ & $14( \pm 9.4)$ & & \\
\hline $\begin{array}{l}\text { Pharmacological } \\
\text { treatment, years (SD) }\end{array}$ & $13( \pm 8.8)$ & & \\
\hline $\begin{array}{l}\text { Number of } \\
\text { hospitalisations (SD) }\end{array}$ & $7.4( \pm 9.8)$ & & \\
\hline $\begin{array}{l}\text { Duration of psychosis, } \\
\text { years since first } \\
\text { episode (SD) }\end{array}$ & $16.4( \pm 10)$ & & \\
\hline $\begin{array}{l}\text { Last hospitalisation, years } \\
\qquad \text { (SD) }\end{array}$ & $7.1( \pm 7.4)$ & & \\
\hline
\end{tabular}


of the proposed study and given oral and written information. The University of Lausanne (School of Medicine) ethical commission approved the protocol and consent procedures. Participation to the study was voluntary and participants were rewarded 25 CHF after the study session.

All the patients were treated with antipsychotic drugs at the time of testing. Fourteen patients received atypical antipsychotic drugs [chlorpromazine equivalents $325.71 \mathrm{mg} /$ day \pm 204.50 , see $(31,32)]$ and one received typical (chlorpromazine equivalent: $500 \mathrm{mg} /$ day) medication. Data were not available for one patient.

\section{Materials and procedure}

Subjects (control and patients) performed a task designed to investigate VSWM processes involved in the encoding of neutral static stimuli composed of eight linear segments [for a detailed description, see (23)]. Stimuli were presented for $2 \mathrm{~s}$ on a screen $\left(27.5^{\circ} \times 20.8^{\circ}\right.$, visual angle). They were composed of eight static line patterns (see Fig. 1). A blank delay lasting $2 \mathrm{~s}$ (white screen) at the end of the stimulus exposure was followed by the recognition slide displaying six patterns (the target pattern and five incorrect patterns) in a $3 \times 2$ grid. Upon the presentation of the discrimination slide, participants were asked to verbalise the number (from 1 to 6) corresponding to the target path. This slide was available as long as required. The task session was composed of 15 stimuli and the subjects did control the start of each new trial.

Five different incorrect patterns were designed to partially match each stimulus. The specific distortions leading to the five incorrect patterns allowed these to be distributed in two different categories: (a) fragmentary: patterns conserving two segments (first two: start patterns; last two: end patterns) or four segments (first and last two: start-end patterns) identical to those of the target trajectory; (b) global: patterns with either the same nine coordinates on the $x$ axis (patterns named rhythm) or on the $y$ axis (patterns named melody) as in the target stimulus. The incorrect patterns (melody and rhythm) in this group approximate the global structure of the target trajectory but they do not have identical segments.

A practice session was performed by all the subjects before beginning the test. Subjects performed trial examples until they demonstrated a clear understanding of the paradigm. Online visual analysis of eye behaviour during the practice and the task sessions was used to ensure that participants actively performed the task. One patient was excluded because of this control analysis.

\section{Oculomotor data acquisition and analysis}

Eye movements' data were collected using an infrared technique (Applied Science Laboratory, H6 model, pupil-corneal reflection tracking, sampling rate $120 \mathrm{~Hz}$, accuracy $0.5^{\circ}$ and resolution of $0.1^{\circ}$ ). Visual stimuli were presented with a projector (resolution of $1280 \times 1024$, with refresh rate of $70 \mathrm{~Hz}$ ). A 9-point calibration procedure was performed after the example, and at the end of the test, to ensure consistency of data collection. The head was not fixed during the tasks, but its position in space was recorded by a magnetic sensor integrated into the helmet (sampling rate of $103 \mathrm{~Hz}$ ). Furthermore, eye movements' data were automatically corrected for head movements.

Eye movement analyses were performed during two specific test phases (see Fig. 1): (a) the stimulus presentation (i.e. encoding) and (b) the discrimination of the correct pattern between the six possibilities. Visual scanning variables included the number of fixations (FX) and the scanning time (ST) in specific regions of interest (ROIs). Eye movement analyses were performed offline with ILAB (33). A fixation was defined as a result of consecutive $x$ and $y$ coordinates that remained within a diameter

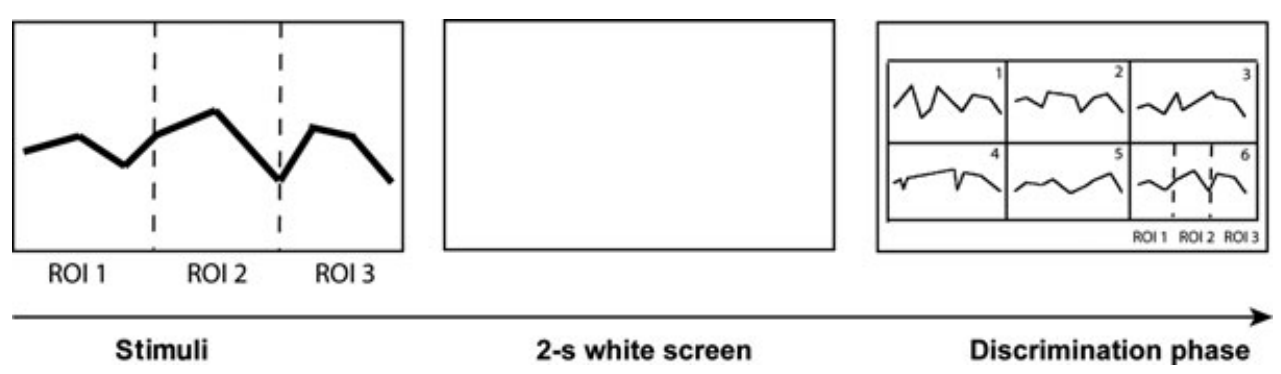

Fig. 1. Illustration of the working memory task design showing the time sequence for each trial. On the left panel are the three stimulus regions of interest (ROIs) (limits not visible during the presentation). On the right is the choice grid with six discrimination ROIs (see 1 to 6 in the discrimination slide, each rectangle represents an ROI). The exploration of the valid path was also analysed from fixations in three specific ROIs (reproducing the stimulus ROIs). 
of $1.5^{\circ}$ of visual angle for at least $150 \mathrm{~ms}(7,11)$. Blinks were identified by a loss of corneal reflection and were excluded from subsequent data analysis, as were gazes directed outside the screen.

Three ROIs were defined to analyse gaze distribution during the stimulus encoding (see Fig. 1). The first three stimuli segments composed of ROI 1, the two central segments, ROI 2, and the three last segments, ROI 3 . For the discrimination phase, we defined six ROIs (discrimination ROI: 1 to 6) corresponding to the six grid cases containing the correct and the five incorrect patterns. Scanning time and FX distribution on the six specific ROIs as well as the distribution of FX on the correct pattern were investigated. The distribution of FX on the correct pattern, during the discrimination phase, was estimated by the same criteria used to define stimulus phase ROIs.

\section{Statistical analysis}

\section{Memory performance}

$T$-test analyses supported group comparisons in task performance. Mixed between- and within-subjects two-way ANOVAs were used to analyse betweengroup (patients vs. controls) difference in incorrect patterns choices. One-way repeated measure ANOVAs were used to investigate within-group differences. Data sphericity was tested using Mauchly's approach. When sphericity was not respected, we employed a Huynh-Feldt correction to rectify the ANOVA results. Chi-squared tests of the distribution of correct choices for the 15 stimuli were performed in each group to assess if all the stimuli were equally difficult. Cohen's $d$ and partial [eta] ${ }^{2}$ were used to estimate effect size.

\section{Eye and head movements}

Stimulus phase. Mann-Whitney (MW) and Wilcoxon tests were used to test the number of fixations between and within the groups. These nonparametric approaches were used because data normality was not confirmed. Horizontal and vertical head movements were assessed from the difference found in each subject for each stimulus between the eye-head movement correlations (MW). The distribution of fixations in the three ROIs of the stimuli was analysed using a mixed two-way ANOVA (groups $\times$ three ROIs as repeated factor). Independent sample $t$-tests were used as post hoc analysis. Cohen's $d$ and partial [eta] ${ }^{2}$ were used to estimate effect size.

Discrimination phase. Mixed between- and within-subjects two-way ANOVAs on FX and ST were used for the following: (a) to compare group visual exploratory patterns in the discrimination ROIs and (b) to compare the exploration of the correct patterns in patients and controls. Specific post hoc comparisons (one-way repeated measure ANOVAs and $t$ tests) were performed to investigate significant global differences in mixed two-way ANOVAs. Cohen's $d$ and partial $[\mathrm{eta}]^{2}$ were used to estimate effect size.

\section{Results}

Memory performance

The distribution of correct choices in each of the 15 trajectories was homogenous in both groups $\left(X^{2}=\right.$ 8.23, $d f=14, p>0.75$ for controls; $X^{2}=2.51$, $d f=14, p>0.99$ for patients). Hence, all the 15 stimuli offered a similar difficulty. Overall, patients made significantly fewer correct choices [average

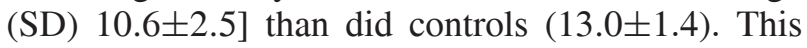
difference was statistically significant $[t(30)=3.281$, $d=1.16, p=0.003$ ].

A mixed two-way ANOVA of error categories (two groups $\times$ five categories as repeated factor) showed a significant group effect [i.e. more wrong choices overall in the patients $F(1,30)=10.765$, partial $[\mathrm{eta}]^{2}=0.264, p=0.003$ ] but no significant error category effect $[F(4,120)=1.652$, partial $\left.[\text { eta }]^{2}=0.05, p=0.166\right]$ or group $\times$ category interaction $\left[F(4,120)=1.252\right.$, partial $[\text { eta }]^{2}=0.04, p=$ $0.293]$.

\section{Oculomotor behaviour}

\section{Stimulus phase (stimulus encoding)}

Controls and patients showed a similar number of fixations during the presentation of the subsequently wrongly identified stimuli (MW $Z=-1.638, p=$ $0.101)$. When exploring the correctly recognised stimuli, however, the patients made more fixations in comparison to controls (MW $Z=-2.507 ; p=$ $0.012)$. Nevertheless, no group exhibited significant differences in number of fixations between correct and incorrect trials (Wilcoxon test: controls $Z=-0.853 ; p=0.394$; patients $Z=-0.894, p=$ 0.371). There was also no significant correlation between the number of fixations and the number of correct responses (controls: Spearman's rho $=0.195$, $p=0.469$; patients: Spearman's rho $=-0.101, p=$ 0.709 ).

Figure 2 shows the number of fixations in each of the three ROIs during the presentation of the subsequently correctly (Fig. 2a) and incorrectly (Fig. 2b) recognised stimuli. The variability appears more important during the exploration of the incorrectly recognised stimuli in both patients and controls. The 


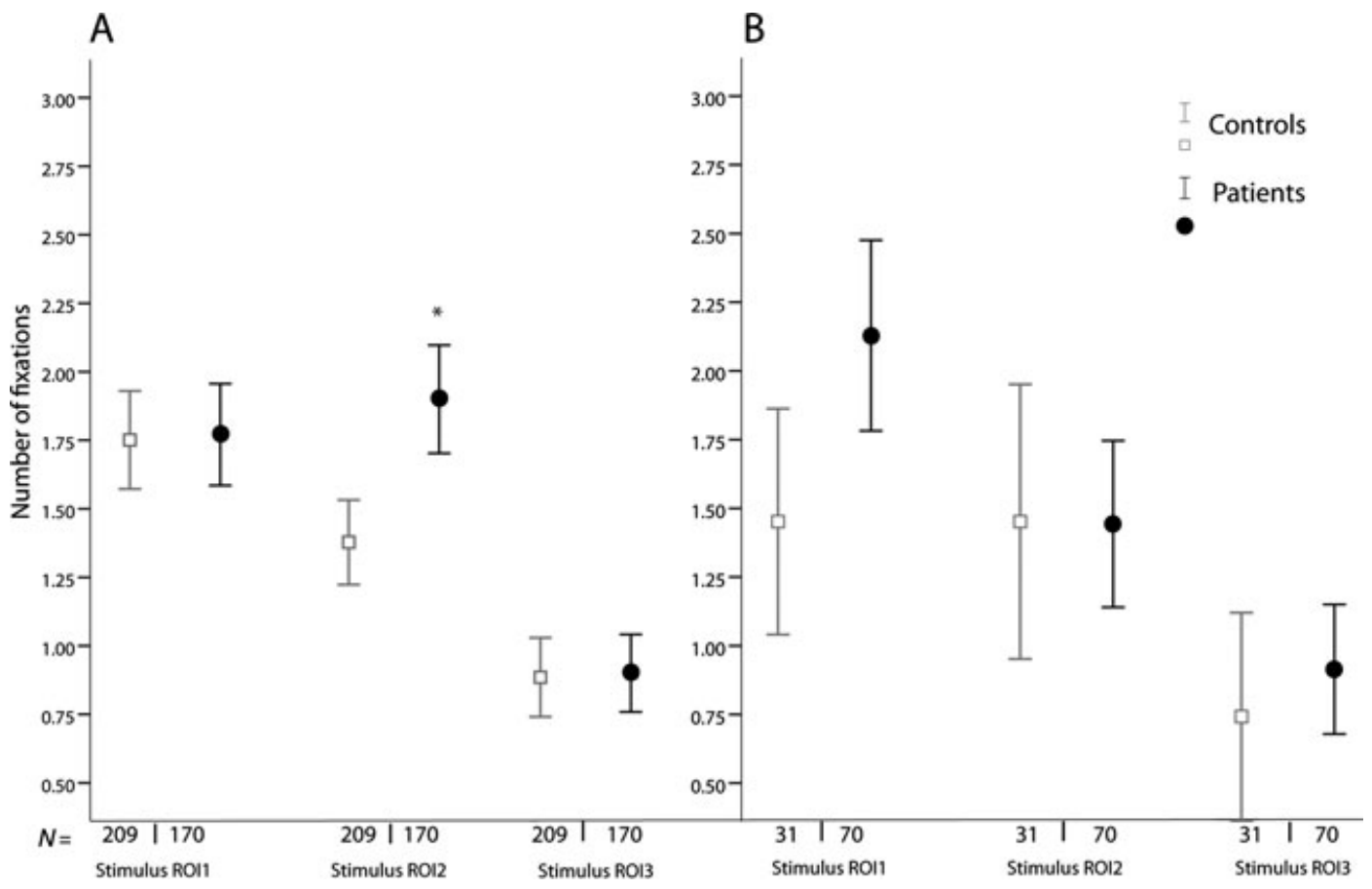

Fig. 2. (a) Mean and error bar (confidence intervals, 95\%) of the number of fixations performed by controls and patients with schizophrenia during the encoding of the correctly recognised stimulus. Patients made less correct recognitions (maximum 240 , grand total $N=170$ ) compared to controls $(N=209)$. (b) Same representation for the visual exploration of the incorrectly recognised stimuli (grand total, patients $N=70$; controls $N=31$ ).

control subjects showed decreased exploration from the left to the right ROIs in the correctly recognised stimuli. This strategy was not evident in the patient group.

The distribution of fixations in the three ROIs of the correctly recognised stimuli was analysed using a mixed two-way ANOVA (groups $\times$ ROIs). This analysis revealed an interaction between group and ROI $\left[F(2,754)=5.143\right.$; partial $[\text { eta }]^{2}=0.013, p=$ 0.006]. A similar interaction was found for scantime $\left[F(1.943,732.576)=3.655\right.$, partial $[\mathrm{eta}]^{2}=$ $0.01, p=0.028]$. Post hoc independent sample $t$ tests showed that patients explored the second (central) stimulus ROI significantly more than did the controls $[\mathrm{FX} t(377)=-4.168, d=0.82, p<0.001$; ST $t(377)=-2.257, d=0.23, p=0.025 ; \quad$ see Fig. 2a]. In contrast, the two groups showed no marked difference in their exploration of the incorrectly recognised stimuli (FX and ST, see Fig. 2b).

Because of a technical problem in head movement output file generation, head movements could only be analysed for 11 patients and 10 controls. This problem had no influence on the correction of eye movements for head movements, automatically performed during the eye movements recording. The average number of positive correlations between eye and head movements during stimuli encoding were similar in the two groups (horizontal axis: $Z=-0.424 ; p=$ 0.672 ; vertical axis: $Z=-0.166 ; p=0.868)$.

\section{Discrimination phase}

Given that response grid durations were not equivalent for all participants, the original data of each subject were related to its total number of fixations and scanning time (\%) in the six ROIs. Visual explorations of the six patterns were analysed separately for the trials with a correct or an incorrect choice. In addition, we analysed whether visual exploration of the correct pattern in correct and incorrect trials was different in the two groups.

Correct trials. A group (control vs. patients) by ROIs (six discrimination ROIs) mixed two-way ANOVA showed a large ROI effect [FX: $F(1$, $3.734)=272.305$, partial $[\text { eta }]^{2}=0.440, p<0.001$; ST: $F(5.696,1456.260)=451.277$, partial $[\text { eta }]^{2}=$ $0.566, p<0.001]$, suggesting the preferential exploration of the correct patterns. There was an incongruent group effect for each of the two visual scanning variables $\left[\mathrm{FX}: F(1,347)=13.830\right.$, partial $[\text { eta }]^{2}=$ $0.038, p<0.001$; ST: $F(1,346)=0.001$, partial $\left.[\text { eta] }]^{2}<0.001, p=0.99\right]$ and no interaction between group and ROI for either visual scanning variables $\left[\mathrm{FX}: F(3.734,1295.798)=1.947\right.$, partial $\left[{ }^{\mathrm{eta}}\right]^{2}=$ 


\section{Cocchi et al.}

(a) Correct trials

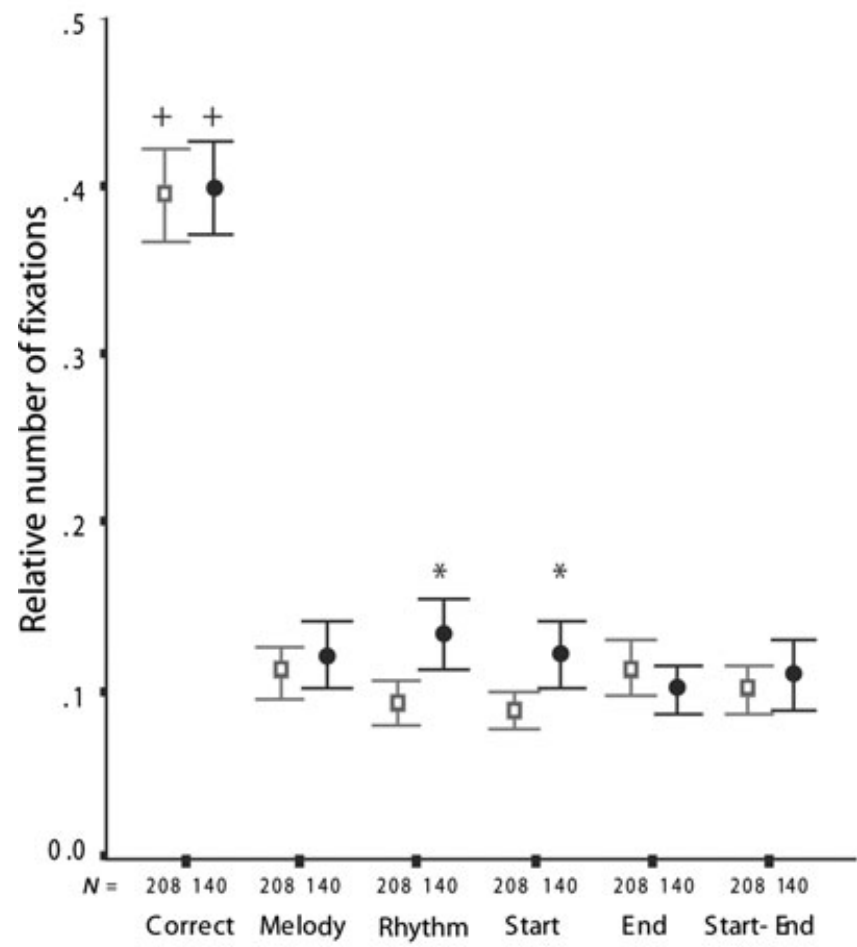

Regions of interest (b) Incorrect trials

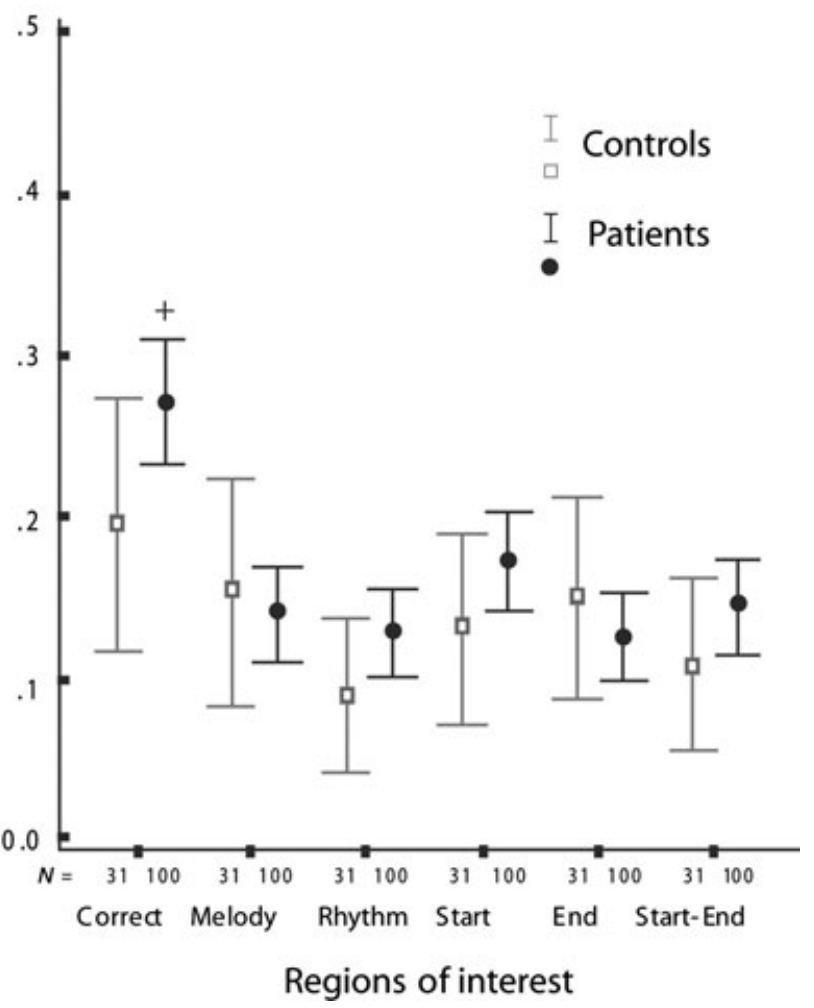

Fig. 3. Mean and confidence intervals (95\%) of the relative number of fixations performed by controls and patients throughout the discrimination phase. $N=$ total number of data for mean computation. '+' indicates significant within-group differences; ' $*$ ' indicates significant between-group differences.

$0.006, p=0.105$; ST: $F(4.209,1456.260)=1.078$, partial [eta] $]^{2}=0.003, p=0.367$; see Fig. 3a].

Within-group one-way repeated measure ANOVAs (six discrimination ROIs as repeated factor) and subsequent post hoc analyses revealed differential exploration of the six discrimination ROIs in the two groups. Indeed, patients and controls explore more the ROI containing the correct pattern in comparison to the other five patterns (paired sample $t$-test: $p<0.01$ for all the five correct vs. incorrect patterns of ROI comparison, FX and ST; see Fig. 3a).

A group (control vs. patients) by ROIs (five 'incorrect' discrimination ROIs) mixed two-way ANOVA revealed a significant interaction between group and ROI factors for the number of fixations $\left[\right.$ FX: $F(3.933,1364.622)=3.223$, partial $[\text { eta }]^{2}=$ $0.009, p=0.013]$. As shown in Fig. 3a, the patients made significantly more fixations on Rhythm ( $t(347)$ $=-3.644, d=0.38, p<0.001)$ and Start $(t(347)=$ $-3.147, d=0.33, p<0.002)$ incorrect patterns.

Incorrect trials. Figure $3 \mathrm{~b}$ suggests that the patient group paid more attention to the correct pattern even though it was not selected.
A group (control vs. patients) by ROIs (six discrimination ROIs as repeated factor) mixed twoway ANOVA revealed the expected ROI effect [FX: $F(4.623,596.403)=6.322$, partial $[\mathrm{eta}]^{2}=$ $0.047, p<0.001$; ST: $F(4.817,621.380)=3.664$, partial $_{\text {eta] }}^{2}=0.028, p=0.003$ ] but failed to show a significant interaction between ROI and group $\left[\mathrm{FX}: F(4.623,596.403)=1.165\right.$, partial $[\mathrm{eta}]^{2}=$ $0.009, p=0.315$; ST: $F(4.817,621.380)=2.048$, partial [eta] ${ }^{2}=0.016, p=0.073$ see Fig. 3b]. However, independent samples $t$-test and effect size exploratory analyses suggest that patients tended to spend more time in scanning the correct patterns than did controls $[t(129)=-2.390, d=0.52, p=$ 0.018]. This is consistent with the within-groups analysis showing that control subjects explored the six discrimination ROIs equally [FX: $F(5,150)$ $=1.450$, partial [eta $]^{2}=0.042, p=0.210 ;$ ST $F(5$, $150)=0.315$, partial [eta $]^{2}=0.01, p=0.903$; see Fig. 3b], whereas patients' exploration was unequally distributed [FX: $F(4.628,458.194)=11.045$, partial $[\text { eta }]^{2}=0.1, p<0.001 ;$ ST $F(4.576,453.061)$ $=11.206$, partial [eta $\left.]^{2}=0.102, p<0.001\right]$. Post hoc 
analyses of the patient group confirmed that the correct pattern was more explored than the five incorrect patterns (paired sample $t$-test: $p<0.001$ for all comparisons of FX and ST; see Fig. 3b for FX).

Visual exploration of the correct pattern during correct and incorrect trials. Mixed two-way ANOVAs (group and three ROIs as repeated factor) revealed no significant effect of the group or ROI and no interaction between these factors for correct and incorrect trials. These results indicate that patients' and controls' explorations of the correct pattern during the discrimination period was similar.

\section{Discussion}

Consistent with our previous research (23), we found that patients made a higher number of incorrect choices than did the control subjects. Detailed analysis of eye movements revealed that, for correct recognitions, control subjects spent most of the encoding phase fixating the leftmost part of the stimulus and very little time on its rightmost part. Because all subjects in this study were native French speakers, this linear exploration from the left to the right side of the stimulus can be related to occidental reading skills (34). During the discrimination phase, controls were mainly fixating on the correct rather than the five incorrect patterns. However, when an incorrect pattern was chosen, the gaze distribution was roughly the same for all six patterns, but there was a high degree of variability suggesting poor stimulus representation.

Analysis of the patients' fixations during the encoding phase showed that, unlike controls, they concentrated particularly on the central ROI when they subsequently recognised the pattern. In contrast, this fixation pattern was absent during the encoding phase of the incorrect trials, suggesting a displacement in attentional focus during encoding of the correct trials. Like controls, however, the patients spent a minimal time in the rightmost ROI during the encoding of all stimuli. During the discrimination phase, the patients differed from the controls in two aspects. Firstly, when selecting the correct patterns, they were particularly attracted by the rhythm and start incorrect patterns. Secondly, they also spent more time inspecting the correct pattern, when subsequently selecting an incorrect one. This specific oculomotor behaviour suggests that the accuracy of the global target representation within VSWM is reduced in patients. Though the patients' visual exploratory strategy differed from that of control subjects, their encoding was sufficient to maintain a relatively high - though diminished - level of accuracy, $70 \%$ compared to a chance level of 1 out of 6 .
In our view, the selective scanning of the central ROI shown by patients during the correct stimuli encoding reveals a specific VSWM encoding strategy. Three main findings suggest that this specific encoding style of the patients is not a direct consequence of a basic oculomotor dysfunction. Firstly, when patients performed a scanning pattern similar to that of the control subjects during correct trials, they made an incorrect response. Secondly, patients explored the three stimulus ROIs at least as controls, in both correct and incorrect trials. Finally, the total number of fixations during stimulus encoding was not associated with the task score, nor was it associated with accuracy in either group.

The majority of experimental designs fix the head during eye movements recording. This artificial constraint prevents the detection of possible compensation of impaired oculomotor processes by head adjustments. As we simultaneously recorded eye and head movements, we observed that head movements during stimulus exploration were not different between the groups, and were not related to impaired performance.

Analysis of eye movements during the discrimination phase showed that correct responses were linked to selective scanning of the correct pattern. For patients, however, significant time was also spent scanning two incorrect patterns: the start patterns, expressing the first part of the stimulus, and the rhythm patterns, approximating its global structure (23). This interest in incorrect patterns with a 'global' structure of the stimulus supports the hypothesis that patients have a general, but imprecise, global internal representation of the stimulus because of the high contribution of early parallel visual encoding processes (e.g. (35)). The assumption that patients relied on a less accurate global representation of the target stimulus is also coherent with the larger number of explorations of the invalid start patterns in comparison to controls.

During the discrimination phase of incorrect trials, control subjects paid little attention to the correct patterns, indicating poor or incomplete stimulus representation. When combined with the large variability in gaze during stimulus encoding and the low number of incorrect choices (13\%), this result suggests that controls' incorrect choices are probably due to a transitory lack of attention or to a miscoding of the target stimulus. In contrast, patients remained attracted by the correct pattern even though they selected another. These findings are also consistent with the assumption that patients' erroneous choices are mainly due to a failure to complete the early global stimuli representation because of limited VSWM resources.

Recent studies show that ageing is accompanied by a significant reduction in VSWM, affecting 
attentional resources and perceptual processes $(29,36$, 37). Therefore, a specific ocular motor behaviour during static stimuli encoding can also be present in healthy aged subjects. Further studies are needed to investigate the specificity of the compensatory encoding mechanisms in schizophrenia, especially in ultra high risk and first episode samples, in order to potentially identify new diagnostic and outcome markers. Understanding particular compensatory processes in subjects with impaired VSWM functions (such as patients with schizophrenia and healthy aged individuals) might also suggest specific intervention in order to improve VSWM abilities.

This study had a number of limitations. Firstly, we could not analyse the relationship between symptoms, visual scanning and VSWM due to limited information about ongoing symptoms at the time of testing. Recent findings suggest that specific symptoms can influence these variables $(13,38)$ - however, the fact that the patients included in this study were in a stable clinical condition probably attenuates the confounding effect of symptoms on our results. Furthermore, all patients were taking antipsychotic medication. Considering the small sample size and the large heterogeneity of medication, we did not consider this variable as a covariate in statistical analysis. In addition, the influence of antipsychotic medication on oculomotor processes $(18,39)$, visual processing (40) and cognitive processes (41) is unclear. Finally, we were not able to match the level of education between controls and patients. However, unpublished behavioural results on healthy subjects $(n=36)$ suggest that this variable is not related to task performance.

In summary, our results confirm the important deficit of patients with schizophrenia in VSWM and suggest that patients try to compensate for reduced VSWM ability by using specific encoding strategies (23). Impaired VSWM ability and specific encoding strategies in schizophrenia do not seem a mere consequence of basic oculomotor dysfunctions.

\section{Acknowledgements}

We thank the patients and control persons who volunteered in this study. The authors especially thank Prof. Pierre Bovet and Dr François Grasset for allowing us to test patients under their care and Dr Pascal Vianin and Jerôme Favrod for their efficient collaboration. The Swiss National Foundation for Scientific Research supported this study (Prof. F. Schenk) 3100A0 - 105765 and (Dr L. Cocchi) PBLAB - 119622. Dr Wood is supported by a Clinical Career Development Award from the NHMRC (Australia). The Robert Bosch Research Institute of Lonay (Switzerland) supported this study by providing technological facilities for eye movement recording and analysis.

\section{References}

1. Pierrot-Deseilligny C, Milea D, Muri RM. Eye movement control by the cerebral cortex. Curr Opin Neurol 2004; 17:17-25.

2. Pierrot-Deseilligny C, Muri RM, Nyffeler T, Milea D. The role of the human dorsolateral prefrontal cortex in ocular motor behavior. Ann N Y Acad Sci 2005;1039:239-251.

3. Pierrot-Deseilligny C, Muri RM, Ploner CJ, Gaymard B, Demeret S, Rivaud-Pechoux S. Decisional role of the dorsolateral prefrontal cortex in ocular motor behaviour. Brain 2003;126:1460-1473.

4. LEE J, PARK S. Working memory impairments in schizophrenia: a meta-analysis. J Abnorm Psychol 2005;114:599-611.

5. Wood SJ, Pantelis C, Proffitt $\mathrm{T}$ et al. Spatial working memory ability is a marker of risk-for-psychosis. Psychol Med 2003;33:1239-1247.

6. Green M, Williams L, Hemsley D. Cognitive theories of delusional formation: the contribution of visual scanpath research. Cognit Neuropsychiatry 2000;5:63-74.

7. Phillips ML, David AS. Visual scan paths are abnormal in deluded schizophrenics. Neuropsychologia 1997;35:99-105.

8. Phillips ML, David AS. Abnormal visual scan paths: a psychophysiological marker of delusions in schizophrenia. Schizophr Res 1998;29:235-245.

9. Williams LM, Loughland CM, Gordon E, Davidson D. Visual scanpaths in schizophrenia: is there a deficit in face recognition? Schizophr Res 1999;40:189-199.

10. Loughland CM, Williams LM, Gordon E. Visual scanpaths to positive and negative facial emotions in an outpatient schizophrenia sample. Schizophr Res 2002;55:159-170.

11. Kojima T, Matsushima E, Ando K et al. Exploratory eye movements and neuropsychological tests in schizophrenic patients. Schizophr Bull 1992;18:85-94.

12. Loughland CM, Williams LM, Gordon E. Schizophrenia and affective disorder show different visual scanning behavior for faces: a trait versus state-based distinction? Biol Psychiatry 2002;52:338-348.

13. Minassian A, Granholm E, Verney S, Perry W. Visual scanning deficits in schizophrenia and their relationship to executive functioning impairment. Schizophr Res 2005;74:69-79.

14. Nakayama H, Morita K, Mori K, Hirai S, Maeda H. Improvement of exploratory eye movements in schizophrenic patients during recovery period. Psychiatry Clin Neurosci 2003;57:169-176.

15. Obayashi S, Matsushima E, Ando H, Ando K, Kojima T, Exploratory eye movements during the Benton Visual Retention Test: characteristics of visual behavior in schizophrenia. Psychiatry Clin Neurosci 2003;57:409-415.

16. MANOR BR, Gordon E, Williams LM et al. Eye movements reflect impaired face processing in patients with schizophrenia. Biol Psychiatry 1999;46:963-969.

17. Bestelmeyer PE, Tatler BW, Phillips LH, Fraser G, Benson PJ, St Clair D. Global visual scanning abnormalities in schizophrenia and bipolar disorder. Schizophr Res 2006;87:212-222.

18. Hutton SB, Huddy V, Barnes TR et al. The relationship between antisaccades, smooth pursuit, and executive dysfunction in first-episode schizophrenia. Biol Psychiatry 2004;56:553-559.

19. Katsanis J, Iacono WG. Clinical, neuropsychological, and brain structural correlates of smooth-pursuit eye tracking 


\section{Oculomotor and working memory processes in schizophrenia}

performance in chronic schizophrenia. J Abnorm Psychol 1991;100:526-534.

20. Litman Re, Hommer DW, Clem T, Ornsteen ML, Ollo C, Pickar D. Correlation of Wisconsin Card Sorting Test performance with eye tracking in schizophrenia. Am J Psychiatry 1991;148:1580-1582.

21. Snitz BE, Curtis CE, Zald DH, Katsanis J, Iacono WG. Neuropsychological and oculomotor correlates of spatial working memory performance in schizophrenia patients and controls. Schizophr Res 1999;38:37-50.

22. Thaker GK, Avila MT, Hong EL, Medoff DR, Ross DE, ADAmi HM. A model of smooth pursuit eye movement deficit associated with the schizophrenia phenotype. Psychophysiology 2003;40:277-284.

23. Cocchi L, Schenk F, Volken H, Bovet P, Parnas J, VIANIN P. Visuo-spatial processing in a dynamic and a static working memory paradigm in schizophrenia. Psychiatry Res 2007;152:129-142.

24. UhlhaAs PJ, Silverstein SM. Perceptual organization in schizophrenia spectrum disorders: empirical research and theoretical implications. Psychol Bull 2005;131:618-632.

25. Butler PD, JAvitT DC. Early-stage visual processing deficits in schizophrenia. Curr Opin Psychiatry 2005;18:151157.

26. Haenschel C, Bittner RA, Haertling F et al. Contribution of impaired early-stage visual processing to working memory dysfunction in adolescents with schizophrenia: a study with event-related potentials and functional magnetic resonance imaging. Arch Gen Psychiatry 2007;64:12291240.

27. Kurylo DD, Pasternak R, Silipo G, Javitt DC, Butler PD. Perceptual organization by proximity and similarity in schizophrenia. Schizophr Res 2007;95:205-214.

28. De Fockert JW, Rees G, Frith CD, Lavie N. The role of working memory in visual selective attention. Science 2001;291:1803-1806.

29. Gazzaley A, Clapp W, Kelley J, McEvoy K, Knight RT, D’Esposito M. Age-related top-down suppression deficit in the early stages of cortical visual memory processing. Proc Natl Acad Sci U S A 2008;105:13122-13126.

30. Sheehan DV, Lecrubier Y, Sheehan KH et al. The Mini-International Neuropsychiatric Interview (M.I.N.I.): the development and validation of a structured diagnostic psychiatric interview for DSM-IV and ICD-10. J Clin Psychiatry 1998;59(Suppl. 20):22-33;quiz 34-57.

31. Woods SW. Chlorpromazine equivalent doses for the newer atypical antipsychotics. J Clin Psychiatry 2003;64:663-667.

32. Leucht S, Wahlbeck K, Hamann J, Kissling W. New generation antipsychotics versus low-potency conventional antipsychotics: a systematic review and meta-analysis. Lancet 2003;361:1581-1589.

33. Gitelman DR. ILAB: a program for postexperimental eye movement analysis. Behav Res Methods Instrum Comput 2002;34:605-612.

34. Liversedge SP, Findlay JM. Saccadic eye movements and cognition. Trends Cogn Sci 2000;4:6-14.

35. Altmann CF, Bulthoff HH, Kourtzi Z. Perceptual organization of local elements into global shapes in the human visual cortex. Curr Biol 2003;13:342-349.

36. Gazzaley A, Cooney JW, Rissman J, D'Esposito M. Top-down suppression deficit underlies working memory impairment in normal aging. Nat Neurosci 2005;8:12981300.

37. Gazzaley A, D'Esposito M. Top-down modulation and normal aging. Ann N Y Acad Sci 2007;1097:67-83.

38. Nishiura S, Morita K, Kurakake K, Igimi H, Maeda H. Characteristics of left and right scanning in schizophrenia patients using exploratory eye movements: comparison with healthy subjects. Psychiatry Clin Neurosci 2007;61:487-494.

39. Gaebel W, Ulrich G, Frick K. Visuomotor performance of schizophrenic patients and normal controls in a picture viewing task. Biol Psychiatry 1987;22:1227-1237.

40. Butler PD, HarkaVy-Friedman JM, Amador XF, GorMAN JM. Backward masking in schizophrenia: relationship to medication status, neuropsychological functioning, and dopamine metabolism. Biol Psychiatry 1996;40:295-298.

41. Daban C, Amado I, Bourdel MC et al. Cognitive dysfunctions in medicated and unmedicated patients with recentonset schizophrenia. J Psychiatr Res 2005;39:391-398.

42. Cocchi L, Bosisio F, Carter O, Wood SJ, Berchtold F, Conus P, Orita A, Debanné M, Schenk F. Visuospatial working memory deficits and visual pursuit impairments are not directly related in schizophrenia. Aust New Zeal J Psychiatr in press. 